\title{
Inhalation Adherence Monitoring Using Smart Electonic Add-on Device
}

\author{
Accuracy evalutaion using simulated real-life test program
}

\author{
Lubomir Gradinarsky and Thomas Lööf \\ Pharmaceutical development \\ AstraZeneca R\&D \\ Mölndal, Sweden \\ Lubomir.Gradinarsky@astrazeneca.com
}

\begin{abstract}
We present an evaluation of the accuracy of an adherence monitoring add-on device (SmartTurbo v2.0, Nexus6 Limited, Auckland, New Zealand) designed to fit a commercial inhalation device (Turbuhaler ${ }^{\circledR}$, dry powder inhaler, AstraZeneca). The evaluation has been based on simulated reallife placebo usage by 11 patients and carried out during a 12 day period. The simulated usage covered low and high inhalation patterns. Of the simulated total 2089 inhalation events 2073 were correctly detected and recorded on the devices' memory. The above indicated an overall accuracy of detection of $99.2 \%$, including possible human errors from the testers. The results confirm that the tested add-on device could successfully be utilized in clinical trials as a reliable replacement of a patient diary report.
\end{abstract}

Keywords - inhalation, adherence, electronic medication monitoring

\section{INTRODUCTION}

A common method for the treatment of asthma and chronic obstructive pulmonary disease (COPD) is use of inhaled medications. These can be prescribed for disease management ("maintenance" therapy) as well as a reliever medication ("as needed" therapy). Patient adherence to their therapy is of key importance for successful healthcare outcomes [1]. Adherence monitoring in research and clinical settings is needed in order to differentiate between poor medication compliance and failed treatment [2]. Commonly, medication usage is recorded by patient self-reports, inhalation device weighting or reading of a dose counter (if such is present at the device). The former method is highly unreliable, and the latter does not provide detailed information on the time of the inhalation events, but only of the total usage. Neither of the above methods is practically suitable for aiding patient compliance in commercial settings.

In this paper, we present a validation study of the accuracy of recording and the performance of a new electronic medication monitoring device (SmartTurbo v2.0, Nexus6 Limited, Auckland, New Zealand). This device is able to automatically follow the patient usage of an inhaler (Turbuhaler®, Dry Powder Inhaler, AstraZeneca) without requiring any active effort from the patient or from medical

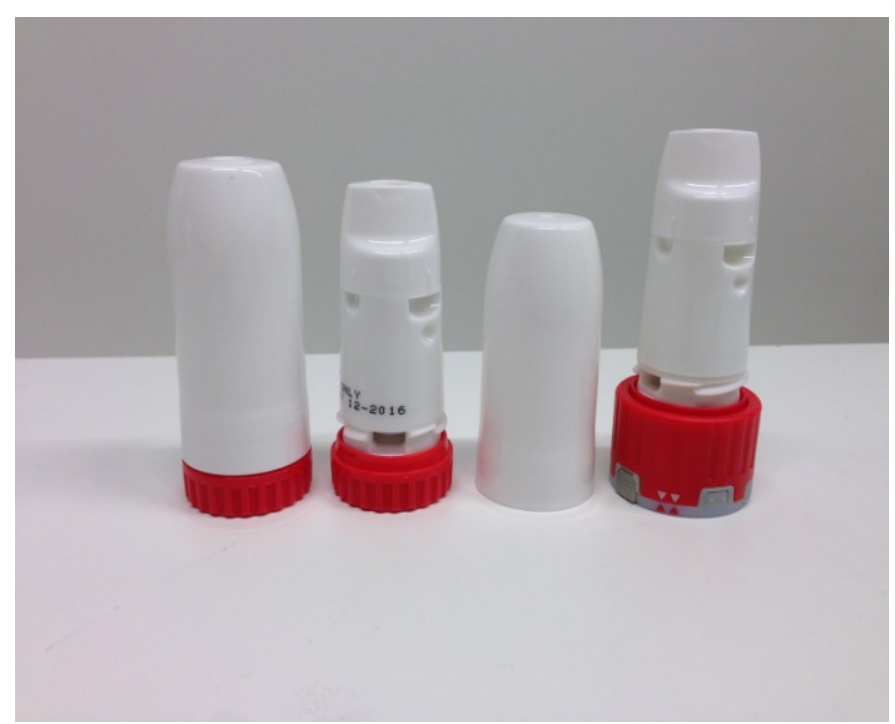

Figure 1. From left to right: A Turbohaler ${ }^{\circledR}$ device with; and without a protection cap; the protection cap; and an open Turbuhaler with a SmartTurbo add-on put on the grip.

personal. We first describe the monitoring device functionality and usage. Then we present the test protocol applied, followed by results and conclusions.

\section{ADHERENCE MONITORING ADD-ON}

The SmartTurbo device tested is designed to fit the Turbuhaler inhaler used in a number of AstraZeneca's inhalation products on the market (Symbicort $\AA$, Pulmicort $\AA$, etc.). The SmartTurbo can be manually placed to the Turbuhaler actuation grip and will not interfere with the inhaler functionality, Fig.1. We tested two version of the Turbuhaler device, which are used with different inhalation products and could be fit in the SmartTurbo. We also tested four different colors of the Turbuhaler grip (the SmartTurbos used had the corresponding color), in order to represent existing products on the market. We thus validate that the adherence monitoring device would work with different Turbuhaler versions and grip colors. 


\section{A. Functionality}

The SmartTurbo adherence monitor detects any consecutive opposite rotations of the Turbuhaler base (red in Fig.1), which corresponds to the inhaler being ready for use by the patient. A sequence of opposite rotations occurring within a minute is interpreted by the internal SmartTurbo software as an inhalation event. The SmartTurbo device also has sensors for detecting that a Turbuhaler is placed or removed from the SmartTurbo and that the protection cap is removed or placed back on the Turbuhaler. In this study we report the accuracy of detection of inhalation events as well as events when the Turbuhaler is replaced from the adherence monitoring add-on.

\section{B. Data Download}

The inhalation actuation events detected by the SmartTurbo are automatically or manually transferred to a mobile phone app via a Bluetooth ${ }^{\circledR}$ Smart technology or to a computer via a USB cable connection. Once downloaded to a mobile phone or to a computer, the data are automatically transferred to the Nexus6 web-based database. From there we retrieve the recorded inhalation data. In this study we used a data transfer to a computer via a USB cable, performed at the end of the study.

\section{Data analyses}

Each of the 11 devices tested was assigned to one patient (tester). The inhalation actuation events recorded by each adherence monitor were compared to the notes in the patient test protocol. For each patient we compared the noted times and the occurrence of the inhalation events. We also looked at events of removal and replacement of the SmartTurbo to the Turbuhaler. We also noted the accuracy of the recorded by the SmartTurbo time. In the results section we present the outcome of these comparison.

\section{TEST PROTOCOL}

In this study we adapted a test protocol representing patient behaviors as defined in [3]. We simulate two types of events spread with different frequency during the test period:

\section{A. Low use}

In order to simulate a "maintenance" or low "as needed" medication usage, we selected to perform two actuation events separated by 10-20 seconds, repeated once again after 10-30 minutes. One low use event will generate 4 inhalations.

\section{B. High use}

In order to simulate a high "as needed" medication usage, we selected to perform eight actuation events separated by 10 20 seconds, repeated once again after 10-30 minutes. One high use event will generate 16 inhalations.

In contrast to the test scheme used in [3] where only one low or one high use per day was adopted (with possible days of no usage in-between), we utilized a more compressed time frame in order to speed-up the study outcome. This more intense testing approach is judged not to have negative impact on the final result outcome due to the fact that the SmartTurbo device records the data immediately. Increasing the frequency of the simulated inhalation events would not introduce any uncertainty on the tested accuracy. Contrary, by applying a
TABLE I. The Utilized Test Protocol

\begin{tabular}{|c|c|c|c|}
\hline Day & When & Event Type & $\begin{array}{l}\text { Number of } \\
\text { inhalations }\end{array}$ \\
\hline \multirow[t]{2}{*}{1} & Before Lunch & Low Use & 4 \\
\hline & After Lunch & Low Use & 4 \\
\hline \multirow[t]{2}{*}{2} & Before Lunch & Low Use & 4 \\
\hline & After Lunch & High Use & 16 \\
\hline \multirow[t]{2}{*}{3} & Before Lunch & Low Use & 4 \\
\hline & After Lunch & Low Use & 4 \\
\hline \multirow[t]{2}{*}{4} & Before Lunch & Low Use & 4 \\
\hline & After Lunch & High Use & 16 \\
\hline \multirow[t]{2}{*}{5} & Before Lunch & Low Use & 4 \\
\hline & After Lunch & High Use & 16 \\
\hline \multirow[t]{2}{*}{6} & Before Lunch & Low Use & 4 \\
\hline & After Lunch & High Use & 16 \\
\hline \multirow[t]{2}{*}{7} & Before Lunch & Low Use & 4 \\
\hline & After Lunch & High Use & 16 \\
\hline \multirow[t]{2}{*}{8} & Before Lunch & Event type 1 & 1 \\
\hline & After Lunch & Event type 2 & 5 \\
\hline \multirow[t]{2}{*}{9} & Before Lunch & Low use & 4 \\
\hline & After Lunch & Low Use & 4 \\
\hline \multirow[t]{3}{*}{10} & Before Lunch & High Use & 16 \\
\hline & After Lunch & Drop test & \\
\hline & & Low use & 4 \\
\hline \multirow[t]{2}{*}{11} & Before Lunch & Low Use & 4 \\
\hline & After Lunch & High Use & 16 \\
\hline \multirow[t]{2}{*}{12} & Before Lunch & Low Use & 4 \\
\hline & After Lunch & High Use & 16 \\
\hline
\end{tabular}

more compressed testing time frame with higher frequency of inhalation events, we could challenge the tested system.

Table I presents the test protocol including a combination of low and high use events for the 12-day test campaign. If all events in the protocol are performed, 190 inhalations would be recorded by each patient. In day 8 we defined events simulating a deviation from the normal Turbuhaler usage. Event type 1 recreated a case when the time interval between the opposite base rotations was more than 1 minute and Event type 2 simulated pattern when the inhaled base was rotated 3 times in opposite directions (instead of 2). The latter should be correctly interpreted as 1 inhalation event. We also tested the SmartTurbo capability of detecting a removal of the Turbuhaler from the SmartTurbo. We introduced the removal event at the start and at the end of the test period. Each tester could optionally on day 10 do a drop test of the Turbuhaler with the SmartTurbo on, followed immediately by a low use event. 
TABLE II. SUMMARY OF THE TEST RESULTS

\begin{tabular}{|c|c|c|c|c|c|c|c|}
\hline $\begin{array}{l}\text { Monitoring } \\
\text { Device/Tester } \\
\end{array}$ & $\begin{array}{l}\text { Turbuhaler } \\
\text { version }\end{array}$ & \begin{tabular}{|l} 
Monitoring Device and \\
Turbuhaler Color
\end{tabular} & $\begin{array}{l}\text { Number of simulated } \\
\text { inhalation events }\end{array}$ & $\begin{array}{l}\text { Missed } \\
\text { Low Use } \\
\end{array}$ & $\begin{array}{l}\text { Missed } \\
\text { High Use } \\
\end{array}$ & $\begin{array}{l}\text { Accuracy of } \\
\text { detection \% }\end{array}$ & \begin{tabular}{|l} 
Internal clock \\
drift sec/day
\end{tabular} \\
\hline 1 & 1 & Brown & 211 & & 1 & 99.5 & +6 \\
\hline 3 & 1 & Brown & 193 & & & 100 & +5 \\
\hline 4 & 1 & Blue Dark & 130 & & & 100 & +4.5 \\
\hline 6 & 2 & White & 203 & & & 100 & +6 \\
\hline 7 & 2 & White & 157 & & & 100 & +5 \\
\hline 8 & 2 & White & 199 & & & 100 & +4.5 \\
\hline 9 & 2 & Red & 201 & & 1 & 99.5 & +5 \\
\hline
\end{tabular}

\section{TEST RESUlts}

Table II summarizes the validation results.

\section{A. Detection of Inhalation events}

Of the simulated in total 2089 inhalation events on the 11 devices used, 2073 have been correctly detected and stored on the devices' memory, indicating an accuracy of detection of $99.2 \%$. Seven devices showed $100 \%$ accuracy. A reason for the missed events could be attributed not only to the SmartTurbo, but also to human errors in simulating the inhalation events or when manually recording the inhalation events. No clear difference in the accuracy of low or high use detection was observed.

\section{B. Inhalation event time}

Before the start of the tests, the internal clocks of all monitoring devices were synchronized to the clock of the computer used for the data readout. At the end of the test period the SmartTurbo clocks were checked again in order to assess their accuracy. The results for the devices (Table II) show positive drifts of 4-6 seconds per day giving a worst case drift of 36.5 minutes per year. This is acceptable accuracy for the purpose of clinical trials and in commercial settings. The SmartTurbo clock is synchronized to the data reading unit clock any time a data download occurs. So when a daily download is to be utilized no noticeable time drift effects will be present.

\section{Inhaler replace and different use detection}

All 22 simulated Turbuhaler replacements (2 for each SmartTurbo) were detected correctly. The simulated usage according to Event type 1 and 2 (described above) were also correctly interpreted by the adherence monitor. No data loss or performance problems were observed after the device drop tests.

\section{CONCLUSIONS AND OUTLOOK}

This paper presents a validation study of the accuracy and reliability of detection of inhalation events as recorded by the SmartTurbo adherence monitoring device. The results provide confidence that such device is fit for purpose and could further more establish itself as standard technology for adherence monitoring in both clinical and commercial settings. The capability of this technology to transfer data in real-time (not tested in this study) will additionally add value to clinical research, to healthcare professionals and to patients. Early detection of patient noncompliance in clinical trials would allow for trial optimization and resource savings. Also nonadherent chronic patients could be recognized and provided with healthcare and motivational support. Real-time data transfer is also of interest for pediatric patients when help from parents/relatives is required.

\section{ACKNOWLEDGMENT}

Sincere thanks to all test volunteers for their participation and for following the test protocol carefully during all test days, including the weekends.

\section{REFERENCES}

[1] L.K. Williams, E.L. Peterson, K. Wells, B.K. Ahmedani, R. Kumar, E.G. Burchard, "Quantifiyng the proportion of severe asthma axacerbations attributable to inhaled corticosteroid nonadherence," J. Allergy Clin. Immunol., vol. 128, pp. 1185-1191, 2004.

[2] L. Osterberg and T. Blaschke, "Adherence to medication," N. Engl. J. Med., vol. 353, pp. 487-497, 2005.

[3] M. Patel, J. Pilcher, A. Chan, K. Perrin, P. Black, R. Beassley "Sixmonth in vitro validation of a metered-deose inhaler electronic monitoring deive: Implications for ashma clinical trial use," J. Clin. Immunol., vol. 130, pp. 1420-1422, 2012. 